\title{
Détermination de l'épaisseur d'une couche mince par transformation de Fourier de la réflectivité du rayonnement $X$
}

\author{
E. Gachon, P. Stemmler, R. Marmoret, J.-M. André* et R. Barchewitz*
}

CEA-DAM/DRIF/SDE, BP. 12, 91680 Bruyères-le-Châtel, France, et, Laboratoire pour I'Utilisation du Rayonnement Électromagnétique, LURE, Université Paris-Sud, 91898 Orsay cedex, France

* Laboratoire de Chimie Physique, Université Pierre et Marie Curie, UMR 7614 du CNRS, 11 rue Pierre et Marie Curie, 75231 Paris cedex 05, France

\begin{abstract}
Résumé: A partir de mesures de réflectivité spéculaire en rayons $X$ rasants, 1 'épaisseur d'une couche mince de carbone déposée sur un substrat de verre a été déterminée. La méthode consiste à effectuer une transformation de Fourier de la réflectivité mesurée en fonction de l'angle d'attaque, ce qui permet d'atteindre fa fonction d'autocorrélation du profil d'indice optique et d'en déduire l'épaisseur de la couche superficielle. L'épaisseur déterminée par cette technique est de $120 \mathrm{~nm}$, pour une épaisseur nominale de $100 \mathrm{~nm}$.
\end{abstract}

\section{INTRODUCTION}

Les couches minces avec des épaisseurs allant du nanomètre à la centaine de nanomètres sont d'un intérêt croissant dans divers domaines de la recherche et de l'industrie. La caractérisation de ces couches, en particulier la détermination de leur épaisseur, est un secteur en plein développement. Dans ce contexte, la réflectométrie rasante du rayonnement $\mathrm{X}$ s'est révélée un outil non destructif particulièrement attractif. La méthode consiste à enregistrer les variations de réflectivité en fonction de l'angle pour un rayonnement $X$ monochromatique. Par suite des interférences entre le rayonnement réfléchi à la surface de l'échantillon et le rayonnement réfléchi à l'interface couche-substrat, la courbe de réflectivité présente des oscillations, appelées franges de Kiessig, qui fournissent une information sur l'épaisseur de la couche. En pratique on cherche à ajuster à la courbe expérimentale, une courbe simulée. Cependant pour que la méthode converge rapidement, il est pratique de disposer d'une valeur approximative initiale pas trop éloignée de la valeur réelle. Cette valeur approximative peut être fournie par transformation de Fourier de la courbe de réflectivité expérimentale.

\section{THEORIE}

Pour un angle d'attaque supérieur à l'angle critique de réflexion totale, la théorie de la diffusion dans le cadre de la première approximation de Born, montre que la réflectivité $\mathrm{R}$ s'exprime en fonction de la dérivée de l'indice de réfraction $\mathrm{n}(\mathrm{z})$ par rapport à la profondeur $\mathrm{z}$ de l'échantillon, comme suit:

$$
R(Q)=\frac{(4 \pi)^{2}}{Q^{4}}\left|\int_{-00}^{+\infty} \frac{\operatorname{dn}(z)}{d z} \exp [i \quad \mathrm{Q} z] d z\right|^{2}
$$

où $Q$ est le vecteur de diffusion:

$$
\mathrm{Q}=\frac{4 \pi \sin \theta}{\lambda}
$$

$\theta$ étant l'angle d'attaque et $\lambda$ la longueur d'onde. En effectuant une transformée de Fourier inverse, on en déduit la fonction d'autocorrélation (FAC) de la dérivée par rapport à $z$ de l'indice de réfraction $\mathrm{n}(\mathrm{z})$ : 


$$
\operatorname{FAC}[n(z)]=\frac{1}{(4 \pi)^{2}} \int_{-\infty}^{+\infty} Q^{4} R(Q) \exp [-i Q z] d Q
$$

\section{MESURES DE LA COURBE DE REFLECTIVITE}

La mesure de la courbe de réflectivité a été effectuée à la longueur d'onde $\lambda=4,13 \mathrm{~A}$ (soit 3 $\mathrm{keV}$ ) a l'aide du dispositif MOGOTOX installé sur la ligne de lumière SB3 de l'anneau de stockage SuperACO du LURE. Ce dispositif comprend successivement: une fente de collimation, un barillet de filtres atténuateurs, un monochromateur équipé en l'occurrence de cristaux de silicium (111) et un gonimètre $\theta-2 \theta$. Le détecteur est un compteur à flux gazeux ( $90 \% \mathrm{Ar}, 10 \%$ méthane) fonctionnant en régime de proportionnalité avec un analyseur monocanal pour éliminer les ordres supérieurs. La courbe de réflectivité de notre système Carbone-Verre pour des angle d'attaque compris entre 0 et 2 degrés est donnée sur la figure 1.

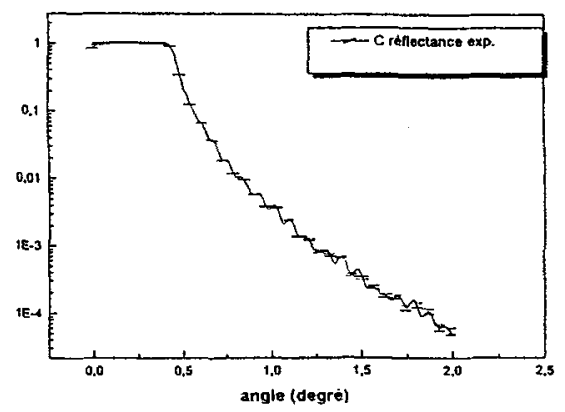

Figure 1: Courbe de réflectivité à $\lambda=4,13 \mathrm{~A}$ du système Carbone-Verre

\section{DETERMINATION DE L' EPAISSEUR DE LA COUCHE DE CARBONE}

Pour un système à j interfaces, la FAC présente $\mathrm{j}(\mathrm{j}-1) / 2$ "raies" associées à chaque couple d'interfaces. Les abcisses de ces "raies" sont les distances entre les interfaces.Pour notre système à 2 interfaces, la FAC présentera donc une "raie" dont la position donne l'épaisseur de la couche. Les courbes expérimentale et théorique de la FAC sont données sur la figure 2. On détecte une "raie" à $1200 \mathrm{~A}$ qui répresente l'épaisseur de la couche. Notons que lors du dépôt une épaisseur de $1000 \mathrm{~A}$ avait été visée.

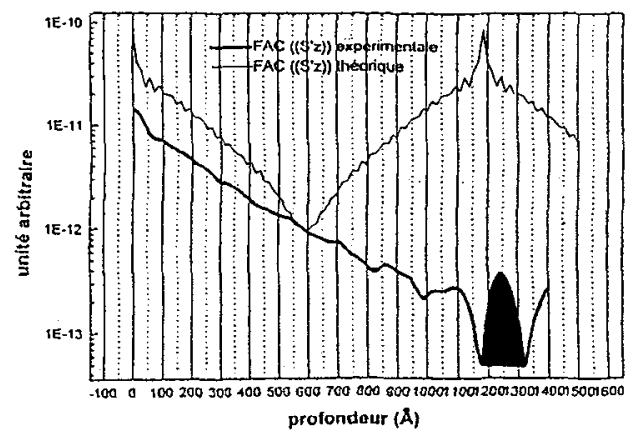

Figure 2: Fonction d'autocorrélation de la dérivée de l'indice pour le système Carbone-Verre 